D) Check for updates

Cite this: DOI: 10.1039/c9fo01298b

\section{A study towards drug discovery for the management of type 2 diabetes mellitus through inhibition of the carbohydrate-hydrolyzing enzymes $\alpha$-amylase and $\alpha$-glucosidase by chalcone derivatives}

\author{
Sónia Rocha, ${ }^{a}$ Adelaide Sousa, ${ }^{a}$ Daniela Ribeiro, ${ }^{a}$ Catarina M. Correia, ${ }^{b}$ \\ Vera L. M. Silva, ID ${ }^{b}$ Clementina M. M. Santos, ${ }^{b, c}$ Artur M. S. Silva, (D) b \\ Alberto N. Araújo, ${ }^{a}$ Eduarda Fernandes (D) a and Marisa Freitas (iD *a
}

Received 18th June 2019 Accepted 24th July 2019

DOI: $10.1039 /$ c9fo01298b

rsc.li/food-function

\begin{abstract}
The inhibition of carbohydrate-hydrolyzing enzymes, $\alpha$-amylase and $\alpha$-glucosidase, is one of the major therapeutic strategies for the treatment of type 2 diabetes mellitus. Chalcones have been recognized for their multiple biological activities, including antidiabetic properties, through unclear mechanisms. In the present work, a panel of chalcones bearing hydroxy, methoxy, methyl, nitro, chloro, fluoro and bromo substituents were evaluated against $\alpha$-amylase and $\alpha$-glucosidase activities, most of them for the first time. The results showed that the substitution patterns and the type of substituents of chalcones influence their inhibitory activity. The presence of hydroxy groups at C-2'- and C-4' of the A ring and at $\mathrm{C}-3$ and $\mathrm{C}-4$ of the $\mathrm{B}$ ring favors the intended effect. Chalcones holding nitro groups and chloro substituents, together with a hydroxy group in the chalcone scaffold, showed strong inhibition of the $\alpha$-glucosidase activity. The present study provides related scaffolds that may serve as the basis for the design and synthesis of new structures in order to obtain the ideal antidiabetic chalcone.
\end{abstract}

\section{Introduction}

Diabetes mellitus (DM) is one of the main worldwide emergencies of the $21^{\text {st }}$ century. The International Diabetes Federation (IDF) reported 425 million people with DM in 2017, and estimated an increase up to 629 million individuals with DM by 2045. ${ }^{1} \mathrm{DM}$ is a chronic metabolic illness characterized by sustained high levels of circulating glucose (hyperglycemia) due to impaired insulin secretion by pancreatic $\beta$ cells, insulin resistance in target tissues, or a combination of both. ${ }^{2}$

Though being classified into three major types, gestational $\mathrm{DM}$, type $1 \mathrm{DM}$ and type $2 \mathrm{DM}$, it is the last one that raises more concern since it accounts for more than $90 \%$ of all cases worldwide. ${ }^{3}$ In type $2 \mathrm{DM}$, insulin levels are insufficient to control postprandial hyperglycemia, which in turn has been

\footnotetext{
${ }^{a}$ LAQV, REQUIMTE, Laboratory of Applied Chemistry, Department of Chemical Sciences, Faculty of Pharmacy, University of Porto, 4050-313 Porto, Portugal. E-mail: marisafreitas@ff.up.pt

${ }^{b}$ QOPNA \& LAQV-REQUIMTE, Department of Chemistry, University of Aveiro, Aveiro, Portugal

${ }^{c}$ Centro de Investigação de Montanha (CIMO) Instituto Politécnico de Bragança, Campus de Santa Apolónia, 5300-253 Bragança, Portugal
}

correlated with the prevalence and incidence of various complications, such as cardiovascular diseases, retinopathy, nephropathy, and neuropathy. ${ }^{4}$ Thus, DM management envisages early intervention and re-establishment of blood glucose levels, thereby decreasing the incidence of complications. ${ }^{5}$ In this regard, one of the therapeutic strategies is to suppress postprandial hyperglycemia by retarding the absorption of glucose through the inhibition of the biocatalyzers involved in carbohydrate digestion, namely $\alpha$-amylase (EC 3.2.1.1) and $\alpha$-glucosidase (EC 3.2.1.20). ${ }^{6}$ During formation of food bolus and swallowing, dietary carbohydrates such as starch start to be breakdown into monosaccharides through cleavage of the $\alpha$-(1,4)-glycosidic bonds by salivary $\alpha$-amylase. This slow process continues in the stomach, but mainly in the upper part of the small bowel where the $\mathrm{pH}$ allows optimal activity of additional pancreatic $\alpha$-amylase secreted into the intestinal lumen and $\alpha$-glucosidase. ${ }^{7}$ As a result, pancreatic $\alpha$-amylase catalyses a more extensive hydrolysis of $\alpha$-(1,4)-glycosidic bonds into maltose, maltotriose, trisaccharides, and other larger oligosaccharides. ${ }^{8}$ Final absorbable monosaccharides, namely glucose, are produced through the activity of the enzyme $\alpha$-glucosidase located in the brush borders of the enterocytes of the jejunum. After $\alpha$-glucosidase action, mono- 
saccharides are transported across the intestinal mucosa to blood vessels and distributed to cells and tissues., ${ }^{9,10}$ Therefore, inhibition of both $\alpha$-amylase and $\alpha$-glucosidase can retard the uptake of dietary carbohydrates and consequently suppress postprandial hyperglycemia, making these enzymes attractive targets for the treatment of type $2 \mathrm{DM}$.

Table 1 Structures and in vitro $\alpha$-amylase and $\alpha$-glucosidase inhibition by chalcones (\% of inhibition at the highest tested concentration, ${ }^{a}$ indicated in superscript)<smiles>[R]c1cc([R])c(C(=O)/C=C/c2c([R])c([R])c([R])c([R])c2[R])c([R])c1</smiles>

$1-28$

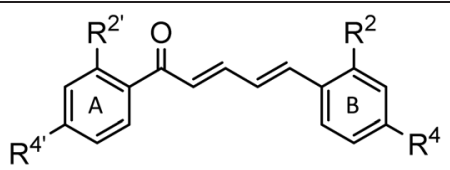

29-41

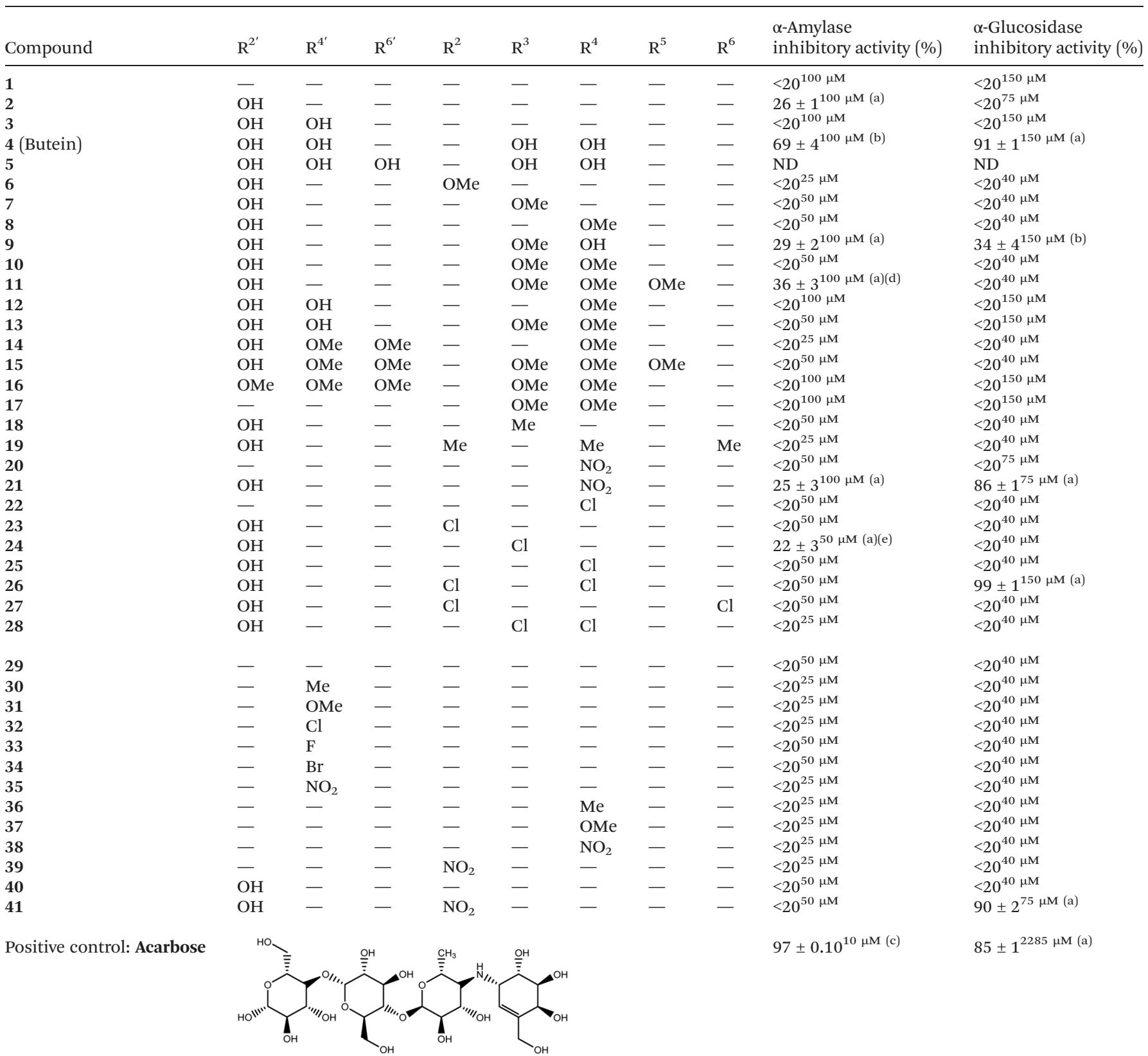

${ }^{a}$ The maximum tested concentrations were established as the concentrations that did not interfere with the assays. ND: Not determined due to interference with the methodology. Same superscript letters indicate that inhibition $\%$ are not statistically different $(p>0.05)$ and different superscript letters indicate that the inhibition $\%$ are statistically different from each other $(p<0.05)$. 
Three $\alpha$-glucosidase inhibitors are currently available in the therapeutic armamentarium to modulate type $2 \mathrm{DM}$, namely acarbose, miglitol, and voglibose. Among these, acarbose also inhibits $\alpha$-amylase activity, being by far the most prescribed drug of this class. $\alpha$-Glucosidase inhibitors are associated with few adverse effects. However, gastrointestinal side effects, including flatulence, soft stools, abdominal discomfort, and diarrhea, have been indicated as the limiting factors. ${ }^{11}$ These adverse effects might occur due to the non-specific inhibition of salivary and pancreatic $\alpha$-amylases, which is correlated with excessive accumulation of undigested carbohydrates in the large intestine. The undigested carbohydrates get fermented by gut microbiota, resulting in intestinal gas production. Alternatively, moderate $\alpha$-amylase inhibition together with high $\alpha$-glucosidase inhibitory activity might overcome such inconvenient effects. ${ }^{12}$ Hence, the discovery of newer and safer $\alpha$-amylase $/ \alpha$-glucosidase inhibitory agents able to modulate type $2 \mathrm{DM}$ has been encouraged.

"Chalcone" is a generic term used to describe compounds with a 1,3-diarylprop-2-en-1-one framework. These compounds, also known as benzalacetophenones or benzylidene acetophenones, are secondary metabolites of terrestrial plants and precursors of flavonoid biosynthesis. ${ }^{13-15}$ The term chalcone is derived from the Greek word "chalkos", meaning "copper/ bronze", underpinning the deep yellow colour owned through strong absorption of the cinnamoyl chromophore within 365-390 nm range, usually bathochromic shifted in derivatives with oxygenated substituents in the phenyl group. ${ }^{16,17}$ Over the last years, increasing attention has been dedicated to chalcones, due to their simple chemistry, ease of synthesis, diversity of substituents, safety, and a vast number of recognized biological activities, including anti-bacterial, anti-parasitic, anti-fungal, anti-viral, anti-cancer, anti-inflammatory, antioxidant, anti-allergic, anti-obesity, anti-hypertensive and antidiabetic activities. ${ }^{15,18,19}$ Several reports even indicate that some chalcones are able to inhibit the enzymes $\alpha$-amylase ${ }^{20-24}$ and $\alpha$-glucosidase..$^{20,22,25-29}$ However, differences found in the experimental conditions regarding this topic, together with the fact that the authors usually constrained the study to a limited number of compounds with decreased rational drug design, make the comparison among the available studies difficult and consequently the establishment of an accurate structure-activity relationship (SAR). With this aim, the inhibitory activity of a panel of twenty-eight chalcones and thirteen chalcone analogues against $\alpha$-amylase and $\alpha$-glucosidase, a total of forty-one structurally related scaffolds (Table 1), most of them being studied here for the first time, is assessed. For this purpose, a microanalysis screening system was applied for testing the type of inhibition of the most active compounds.

\section{Materials and methods}

\subsection{Chemicals}

The following reagents were obtained from Sigma-Aldrich Co. LLC (St Louis, MO): acarbose, $\alpha$-amylase from porcine pan- creas, $\alpha$-glucosidase from Saccharomyces cerevisiae, 2-chloro- $p$ nitrophenyl- $\alpha$-D-maltotrioside (CNPG3), dimethyl sulfoxide (DMSO), $\quad p$-nitrophenyl- $\alpha$-D-glucopyranoside ( $p$ NPG), and sodium hydrogen phosphate. Compounds 1-3, 7-13, 16, and 17 were obtained from Indofine Chemical Company, Inc. (Hillsborough, NJ). Compounds 4 and 5 were obtained from Extrasynthese (Z.I Lyon Nord). Compounds 6, 14, 15, and 18-41 were synthesized as previously described. ${ }^{30-33}$

All compounds were dissolved in DMSO at concentrations high enough to ensure low final $\%(<4.76 \% \mathrm{v} / \mathrm{v})$ of the solvent along enzymatic assays.

\subsection{In vitro $\alpha$-amylase inhibition assay}

The $\alpha$-amylase activity was measured using a method previously reported by Proença et $a{ }^{34}{ }^{34}$ with slight modifications. All concentrations refer to the final volume of the reactional mixture. In a 96-well plate, $0.1 \mathrm{U} \mathrm{mL}^{-1} \alpha$-amylase, dissolved in $20 \mathrm{mM}$ phosphate buffer ( $\mathrm{pH}$ 6.8), was preincubated at $37^{\circ} \mathrm{C}$ for 10 minutes with the chalcones under study in concentrations up to $100 \mu \mathrm{M}$. Acarbose $(0-10 \mu \mathrm{M})$ was used as positive control. After attainment of thermal equilibrium, $500 \mu \mathrm{M}$ of CNPG3 substrate in phosphate buffer was added, followed by another incubation, at $37^{\circ} \mathrm{C}$ for 30 minutes. For this, a microplate reader (Synergy HT, BIOTEK) was used with the wavelength set to $405 \mathrm{~nm}$. Then, the derivative values of the absorbance versus time plots, in the interval of 5 to 15 minutes, were calculated and used for further processing. The obtained results were expressed as the mean \% inhibition of $\alpha$-amylase activity, and represent at least three independent experiments. The results were always compared with the positive control, acarbose.

2.2.1 Study of the $\alpha$-amylase inhibition type. The inhibition mechanism of $\alpha$-amylase was identified for the most active compounds in the previous screening study, namely chalcone 4 (butein) $(0-50 \mu \mathrm{M})$ and the positive control acarbose $(0-0.75 \mu \mathrm{M})$. Therefore, the compounds were pre-incubated with $0.1 \mathrm{U} \mathrm{mL}^{-1} \alpha$-amylase, dissolved in $20 \mathrm{mM}$ phosphate buffer ( $\mathrm{pH}$ 6.8), and incubated for 10 minutes at $37^{\circ} \mathrm{C}$. In sequence, the substrate CNPG3 was added at three different concentrations, 250, 500 and $1000 \mu \mathrm{M}$, and the absorbance of the reaction mixture at the wavelength of $405 \mathrm{~nm}$ was followed for 30 minutes at $37^{\circ} \mathrm{C}$. Then, the derivative values of the absorbance versus time plots in the interval of 5 to 15 minutes were calculated and used for further processing.

The generalized Michaelis-Menten eqn (1) describing the kinetics and each one of its simplifications regarding the different types of inhibitions were used as models for nonlinear regression of the results obtained from at least five independent experiments:

$$
V_{\text {inic }}=\frac{V_{\text {máx }}(S)}{K_{\mathrm{m}}\left(1+\frac{|I|}{K_{\mathrm{ic}}}\right)+(S)\left(1+\frac{|I|}{K_{\mathrm{iu}}}\right)}
$$

where $V_{\text {inic }}=$ initial velocity of formation of absorbing CNP in $\Delta$ Absorbance per minute, $V_{\max }=$ maximum achievable velocity 
when for the $0.1 \mathrm{U} \mathrm{mL}^{-1}$ of enzyme had used all the catalytic sites and is saturated by the substrate, $S=$ CNPG3 concentration in $\mathrm{mM}, K_{\mathrm{m}}=$ Michaelis-Menten constant in $\mathrm{mM}, K_{\mathrm{ic}}=$ inhibitor dissociation constant of enzyme inhibitor expressed in $\mu \mathrm{M}^{-1}, K_{\mathrm{iu}}=$ inhibitor dissociation constant of enzyme-substrate-inhibitor complex expressed in $\mu \mathrm{M}^{-1}$.

Data fitting was performed in an Excel Microsoft Office ${ }^{\mathrm{TM}}$ spreadsheet using the Solver ${ }^{\mathrm{TM}}$ supplement according to Bezerra et $a l .{ }^{35}$ and Dias et $a l .{ }^{36}$ For each tested condition, the equation parameters were estimated by Solver ${ }^{\mathrm{TM}}$. The obtained values for the parameters of the simplest model (without inhibition) were used as initial guesses and for the other types of inhibition, competitive, non-competitive, uncompetitive, and finally the more complex model, mixed inhibition. The actual mechanism of inhibition was established by comparison between models, applying the extra sum-of-square $F$ test $^{37}$ and the Akaike information criterion (AIC) test. $^{38}$ In order to determine the error of the kinetic constants values, the Jackknife procedure was applied, which consisted of the calculation of standard deviation of all estimations made by Solver ${ }^{\mathrm{TM}}$ when each experimental data point was removed from the initial set. The analyses of the corresponding Lineweaver-Burk plots for each concentration of the inhibitor and substrate were performed additionally.

\subsection{In vitro $\alpha$-glucosidase inhibition assay}

$\alpha$-Glucosidase activity was measured using a method previously reported by Proença et $a .^{39}$ with slight modifications. All concentrations refer to the final volume of the reactional mixture. In a 96-well plate, $0.04 \mathrm{U} \mathrm{mL}^{-1}$ of $\alpha$-glucosidase was dissolved in $100 \mathrm{mM}$ phosphate buffer $(\mathrm{pH}$ 6.8) and was pre-incubated with the chalcones under study in concentrations up to $150 \mu \mathrm{M}$ for 5 minutes at $37^{\circ} \mathrm{C}$. Acarbose (0-2285 $\mu \mathrm{M})$ was herein also used as positive control. The catalytic reaction started with the addition of the phosphate buffered solution containing $600 \mu \mathrm{M}$ of $p$ NPG substrate, and the reaction mixture was incubated at $37{ }^{\circ} \mathrm{C}$ for 30 minutes. The enzymatic reaction was monitored spectrophotometrically at $405 \mathrm{~nm}$ by measuring the resultant yellow color formed. Then, the derivative values of the absorbance versus time plots in the interval of 5 to 15 minutes were calculated and used for further processing. The obtained results were expressed as the mean $\%$ inhibition of $\alpha$-glucosidase activity and represent at least three independent experiments. The results were always compared with the positive control, acarbose.

2.3.1 Study of the $\alpha$-glucosidase inhibition type. The types of $\alpha$-glucosidase inhibition were studied for the most active chalcones, 4 (butein) (0-75 $\mu \mathrm{M}), 21(0-45 \mu \mathrm{M}), 26(0-65 \mu \mathrm{M})$, $41(0-40 \mu \mathrm{M})$, and the positive control, acarbose $(0-2285 \mu \mathrm{M})$. In a 96-well plate, $0.04 \mathrm{U} \mathrm{mL}^{-1} \alpha$-glucosidase, dissolved in $100 \mathrm{mM}$ phosphate buffer ( $\mathrm{pH}$ 6.8), was pre-incubated with the compounds under study for 5 minutes at $37{ }^{\circ} \mathrm{C}$. Then, the substrate $p$ NPG was added at three different concentrations, 300, 600 and $1200 \mu \mathrm{M}$, and the reaction mixture was incubated at $37^{\circ} \mathrm{C}$ for 30 minutes. Then, the derivative values of the absor- bance versus time plots in interval of 5 to 15 minutes were calculated and used for further processing.

The types of inhibition of $\alpha$-amylase were studied as mentioned in the section 2.2.1. and the results represent at least five independent experiments.

\subsection{Statistical analysis}

The results of the in vitro inhibitory activities of the chalcones against pancreatic $\alpha$-amylase and $\alpha$-glucosidase are expressed as mean \pm standard error of the mean (SEM). A statistical comparison between the active chalcones was performed using one-way analysis of variance (ANOVA). Differences among the groups were compared by Tukey test, with a $p$ value $<0.05$ considered statistically significant. All the statistical analyses were performed using GraphPad Prism ${ }^{\mathrm{TM}}$ (version 5.0; GraphPad Software).

The type of inhibition was established by comparison among the models using the Solver ${ }^{\mathrm{TM}}$ and by applying the extra sum-of-square F test and AIC test. ANOVA was applied to evaluate the precision of the method.

\section{Results}

\subsection{In vitro $\alpha$-amylase inhibition}

The inhibitory effects of compounds 1-41 and the positive control, acarbose, against pancreatic $\alpha$-amylase are shown in Table 1 . The compounds were divided as chalcones 1-28, with the 1,3-diarylprop-2-en-1-one framework, and chalcone analogues 29-41, known as cinnamylideneacetophenones, which hold two double bonds linking the A and B rings. In the first set of compounds (1-28), one is unsubstituted (1), four are hydroxylated (2-5), ten are hydroxylated and methoxylated (6-15), two are only methoxylated $(\mathbf{1 6}, \mathbf{1 7})$, two display methyl substituents $(\mathbf{1 8}, \mathbf{1 9})$, two display nitro groups $(\mathbf{2 0}, \mathbf{2 1})$ and seven possess chloro substituents (22-28). Among the chalcone analogues (29-41), one is unsubstituted (29), eleven present a single substitution, including methyl (30, 36), methoxy $(31,37)$, chloro (32), fluoro (33), bromo (34), nitro (35, $38,39)$ and hydroxy $(\mathbf{4 0})$. Compound (41) is di-substituted with a hydroxy group and a nitro group.

Among the hydroxylated chalcones (2-5), chalcone 4 (butein) with two hydroxy groups at $\mathrm{C}-2$ ' and $\mathrm{C}-4$ ' of the A ring and two at C-3 and C-4 of the B ring exhibited the highest inhibitory activity of $69 \pm 4 \%$ at the highest tested concentration of $100 \mu \mathrm{M}$. These results indicate that these substituents are important for the intended effect on $\alpha$-amylase. Chalcone 2 $(26 \pm 1 \%$, at $100 \mu \mathrm{M})$ with only one $2^{\prime}$-hydroxy group at the A ring also showed slight activity, though additional presence of this substituent at C-4' hydroxy (compound 3) cancels the effect. Unfortunately, it was not possible to evaluate compound 5 due to a methodological interference. During the assays, an irregular slope in the absorbance versus time plots was observed which can be due to the possible cyclisation of the $6^{\prime}$-hydroxy group ${ }^{40}$ that altered the absorbance spectrum and, consequently, the behavior of the inhibition curve. 

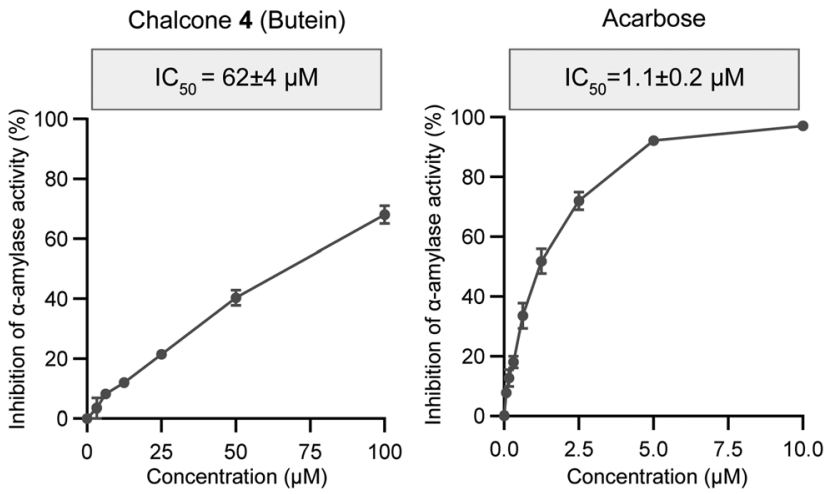

Fig. $1 \alpha$-Amylase inhibition by chalcone 4 (butein) and the positive control, acarbose. Each value represents mean \pm SEM of at least three experiments.

On comparison, the positive control acarbose $\left(\mathrm{IC}_{50}=\right.$ $1.1 \pm 0.2 \mu \mathrm{M}$ ) (Fig. 1) had a substantially higher activity than chalcone 4 (butein) ( $\left.\mathrm{IC}_{50}=62 \pm 4 \mu \mathrm{M}\right)$. Thus, chalcone 4 (butein) shows a moderate inhibition against $\alpha$-amylase when compared with the positive control, acarbose.

Within the hydroxylated and/or methoxylated derivatives (6-17), low inhibitory activities were observed such as $29 \pm 2 \%$ for $100 \mu \mathrm{M}$ chalcone 9 and $36 \pm 3 \%$ for $100 \mu \mathrm{M}$ chalcone 11. In general, methoxy substituents seem to be disadvantageous for the inhibitory activity. Similarly, the studied chalcones with methyl substituents (18 and 19) were not able to inhibit $\alpha$-amylase at the highest tested concentrations.

Chalcones holding nitro groups (20 and 21) also showed reduced activity. The last, holding a 2'-hydroxy group at the A ring and a C-4 nitro group at the $\mathrm{B}$ ring, showed the highest effect of $25 \pm 3 \%$, at $100 \mu \mathrm{M}$.

Chalcones with chloro substituents (22-28) presented a low $\alpha$-amylase inhibition. Among these seven compounds, only chalcone $24(22 \pm 3 \%$ at $50 \mu \mathrm{M})$ which has a 2'-hydroxy group at the A ring and a 3-chloro in the B ring had above $20 \%$ activity.

The enone functionality of chalcones underpins the inhibitory activity. In fact, compounds holding two double bonds linking the A and B rings, the chalcone analogues (29-41), were not able to inhibit $\alpha$-amylase activity, regardless of the substituent group, up to the highest tested concentration.

3.1.1 Type of $\alpha$-amylase inhibition. The type of inhibition mechanisms of chalcone 4 (butein), the most active member of all the chalcones, and the positive control acarbose were deduced from the statistical evaluations of the experimental data fitting to the corresponding Michaelis-Menten kinetics model and Lineweaver-Burk plots. Meanwhile, one-factor ANOVA analysis of the raw experimental data showed a precision better than 1.6 and $1.4 \Delta$ absorbance per minute for chalcone 4 and acarbose, respectively, as calculated from the within-groups mean square. The same statistical test also evidenced the method adequacy for the proposed objective since $F$ values of 35 and 60, higher than the $F$ critical values (2.0 and 1.9, respectively), indicating the clear inhibitory
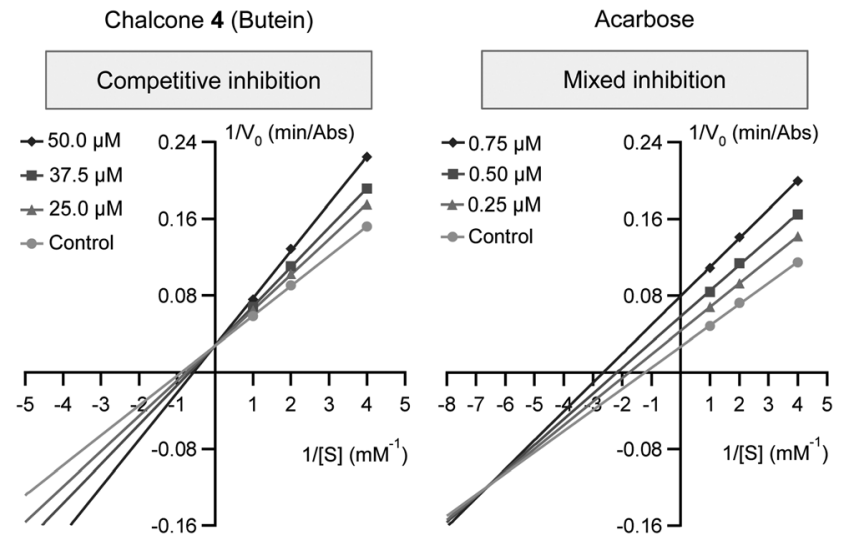

Fig. 2 Lineweaver-Burk plots of $\alpha$-amylase inhibition by chalcone 4 (butein) and acarbose, the positive control.

effect of both the compounds on the enzymatic activity. As depicted in Fig. 2, chalcone 4 (butein) behaved as a competitive inhibitor, since the $K_{\mathrm{m}}$ value increased and $V_{\max }$ value remained constant with increasing concentrations of the compound. A mixed inhibition mechanism was devised for acarbose once to the increase in concentration corresponded decreasing values of $K_{\mathrm{m}}$ and $V_{\max }$. In both cases, the indicated kinetic model for inhibition was the best fitted, thus, providing the lowest sum squared residuals after iterative non-linear regression using the Solver ${ }^{\mathrm{TM}}$ supplement. The results for the type of inhibition and the kinetic constants values $\left(V_{\max }, K_{\mathrm{m}}, K_{\mathrm{ic}}\right.$ and/or $\left.K_{\mathrm{iu}}\right)$ are summarized in Table 2.

\subsection{In vitro $\alpha$-glucosidase inhibition}

Results presented in Table 1 show some chalcones with effective inhibitory activity on $\alpha$-glucosidase from Saccharomyces cerevisiae. The most potent inhibitors were chalcone 4 (butein), chalcone 21, chalcone 26 and chalcone analogue 41, with $\mathrm{IC}_{50}$ values of $21 \pm 2 \mu \mathrm{M}, 53 \pm 1 \mu \mathrm{M}, 87 \pm 3 \mu \mathrm{M}$, and $41 \pm 1 \mu \mathrm{M}$, respectively (Fig. 3).

Among the hydroxylated compounds (2-5), chalcone 4 (butein), bearing hydroxy groups at $\mathrm{C}-2^{\prime}$ and $\mathrm{C}-4^{\prime}$ of the A ring and $\mathrm{C}-3$ and $\mathrm{C}-4$ of the $\mathrm{B}$ ring was the most active compound with an $\mathrm{IC}_{50}$ value nearly seventeen times lower than the $\mathrm{IC}_{50}$ value of acarbose, the positive control $\left(\mathrm{IC}_{50}=357 \pm 25 \mu \mathrm{M}\right)$ (Fig. 3). Chalcone 4 (butein) shows a potent inhibition of $\alpha$-glucosidase, when compared with the positive control, acarbose. Chalcone 2, with a 2 '-hydroxy group at the A ring, and chalcone 3 with hydroxy groups at $\mathrm{C}-2^{\prime}$ and $\mathrm{C}-4^{\prime}$ of the A ring, both without substituents at the B ring, showed no inhibitory activity. Considering the hydroxylated and methoxylated chalcones (6-15) and the methoxylated chalcones (16 and 17), only chalcone 9 bearing a 2'-hydroxy group at the A ring, a 3-methoxy substituent, and a 4-hydroxy group at the $\mathrm{B}$ ring showed slight inhibitory activity of $34 \pm 4 \%$, at $150 \mu \mathrm{M}$.

Methyl substituted compounds (18 and 19) were inactive up to the highest tested concentration. 
Table 2 Type of inhibition (using Solver ${ }^{\mathrm{TM}}$ supplement) of chalcone 4 (butein) and acarbose against $\alpha$-amylase activity, and chalcones 4 (butein), 21,26 , chalcone analogue 41 and acarbose against $\alpha$-glucosidase activity and respective kinetic parameters values: $V_{\max }, K_{\mathrm{m}}, K_{\mathrm{ic}}$ and $K_{\mathrm{iu}}(\mathrm{mean} \pm$ SEM)

\begin{tabular}{|c|c|c|c|c|c|}
\hline Compound & Type of inhibition & $V_{\max }$ & $K_{\mathrm{m}}$ & $K_{\mathrm{ic}}$ & $K_{\mathrm{iu}}$ \\
\hline \multicolumn{6}{|c|}{$\alpha$-Amylase activity } \\
\hline 4 (Butein) & Competitive & $35 \pm 1$ & $1.17 \pm 0.03$ & $88 \pm 2$ & - \\
\hline Acarbose & Mixed & $41 \pm 1$ & $0.94 \pm 0.02$ & $2.6 \pm 0.2$ & $0.37 \pm 0.01$ \\
\hline \multicolumn{6}{|c|}{$\alpha$-Glucosidase activity } \\
\hline 4 (Butein) & Competitive & $97 \pm 2$ & $0.91 \pm 0.04$ & $13.7 \pm 0.4$ & - \\
\hline 21 & Non-competitive & $208 \pm 5$ & $2.9 \pm 0.1$ & $103 \pm 2$ & $103 \pm 2$ \\
\hline 26 & Non-competitive & $125 \pm 5$ & $1.49 \pm 0.09$ & $138 \pm 4$ & $138 \pm 4$ \\
\hline Analogue 41 & Non-competitive & $139 \pm 3$ & $1.51 \pm 0.07$ & $51 \pm 1$ & $51 \pm 1$ \\
\hline Acarbose & Competitive & $112 \pm 2$ & $1.49 \pm 0.05$ & $246 \pm 5$ & - \\
\hline
\end{tabular}
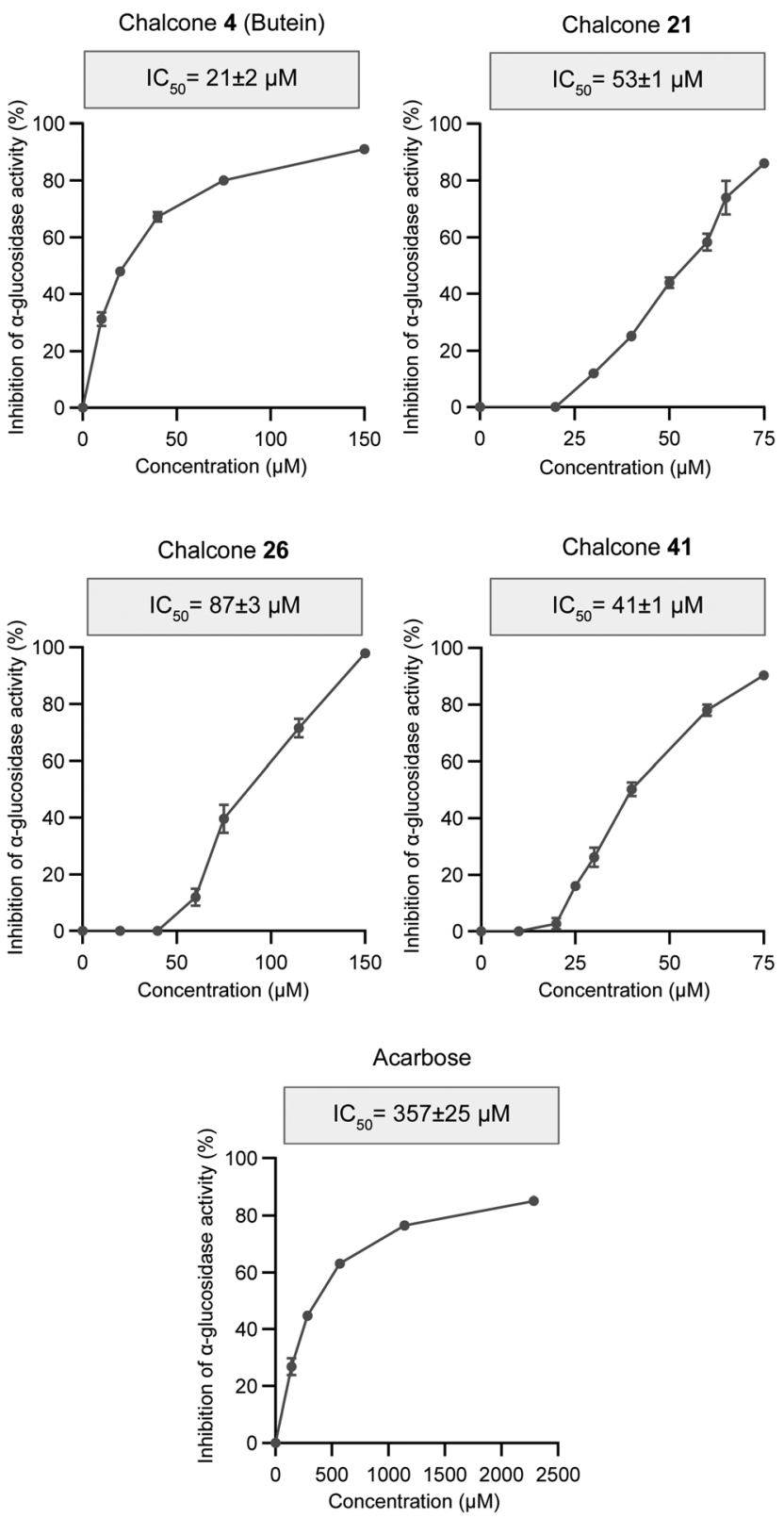

Fig. $3 \alpha$-Glucosidase inhibition by chalcones 4 (butein), 21, and 26, chalcone analogue 41 and acarbose, the positive control. Each value represents mean \pm SEM of at least three experiments.
Within chalcones holding nitro groups (20 and 21), chalcone 21, bearing a 2'-hydroxy group at the A ring and a 4-nitro group at the $\mathrm{B}$ ring, showed almost seven times more activity $\left(\mathrm{IC}_{50}=53 \pm 1 \mu \mathrm{M}\right)$ than the positive control, acarbose $\left(\mathrm{IC}_{50}=\right.$ $357 \pm 25 \mu \mathrm{M})$ (Fig. 3).

Among the chlorinated chalcones (22-28), it is possible to conclude that chalcone 26 bearing a 2'-hydroxy group at the $\mathrm{A}$ ring and the chloro substituents at $\mathrm{C}-2$ and $\mathrm{C}-4$ of the $\mathrm{B}$ ring was the most active compound with an $\mathrm{IC}_{50}$ value of $87 \pm 3 \mu \mathrm{M}$ which is nearly four times lower than acarbose (Fig. 3). Chalcones 22-25, 27, and 28 had no activity against this enzyme at the highest tested concentration.

Among the chalcone analogues (29-41), the last derivative (41) with a 2'-hydroxy group at the A ring and a 2-nitro group at the $\mathrm{B}$ ring was almost nine times more active with an $\mathrm{IC}_{50}$ value of $41 \pm 1 \mu \mathrm{M}$ when compared with the positive control acarbose (Fig. 3). Hence, while the group enone, herein with an extra double bond added, seems less important, the simultaneous presence of the nitro group at C-2 together with the hydroxy group at C-2' seems to be essential for the inhibitory activity.

3.2.1 Type of $\alpha$-glucosidase inhibition. The inhibition mechanisms of the most active compounds, chalcones 4 (butein), 21, and 26, chalcone analogue 41, and acarbose against $\alpha$-glucosidase were assessed by an approach similar to the one already described for $\alpha$-amylase. The one-factor ANOVA analysis validated the experimental method used and showed acceptable precisions ranging from $1.9 \Delta$ absorbance per minute up to $5.5 \Delta$ absorbance per minute, respectively, for acarbose and chalcone $\mathbf{2 6}$ along assays. The same test also evidenced the clear inhibition effect after analysis of mean squares between groups. Fig. 4 shows the Lineweaver-Burk plots of $\alpha$-glucosidase activity in the presence of the selected compounds. Chalcone 4 (butein) and acarbose behaved as competitive inhibitors of $\alpha$-glucosidase. The $V_{\max }$ value remained constant regardless of the inhibitor concentration, but higher concentrations of the inhibitors led to increasing $K_{\mathrm{m}}$ values. Chalcones 21, 26, and 41 underpinned non-competitive inhibition plots with constant $K_{\mathrm{m}}$ values but successive lower $V_{\max }$ values. Corresponding kinetic parameters, obtained from nonlinear regression of respective inhibition theoretical models are shown in Table 2. 

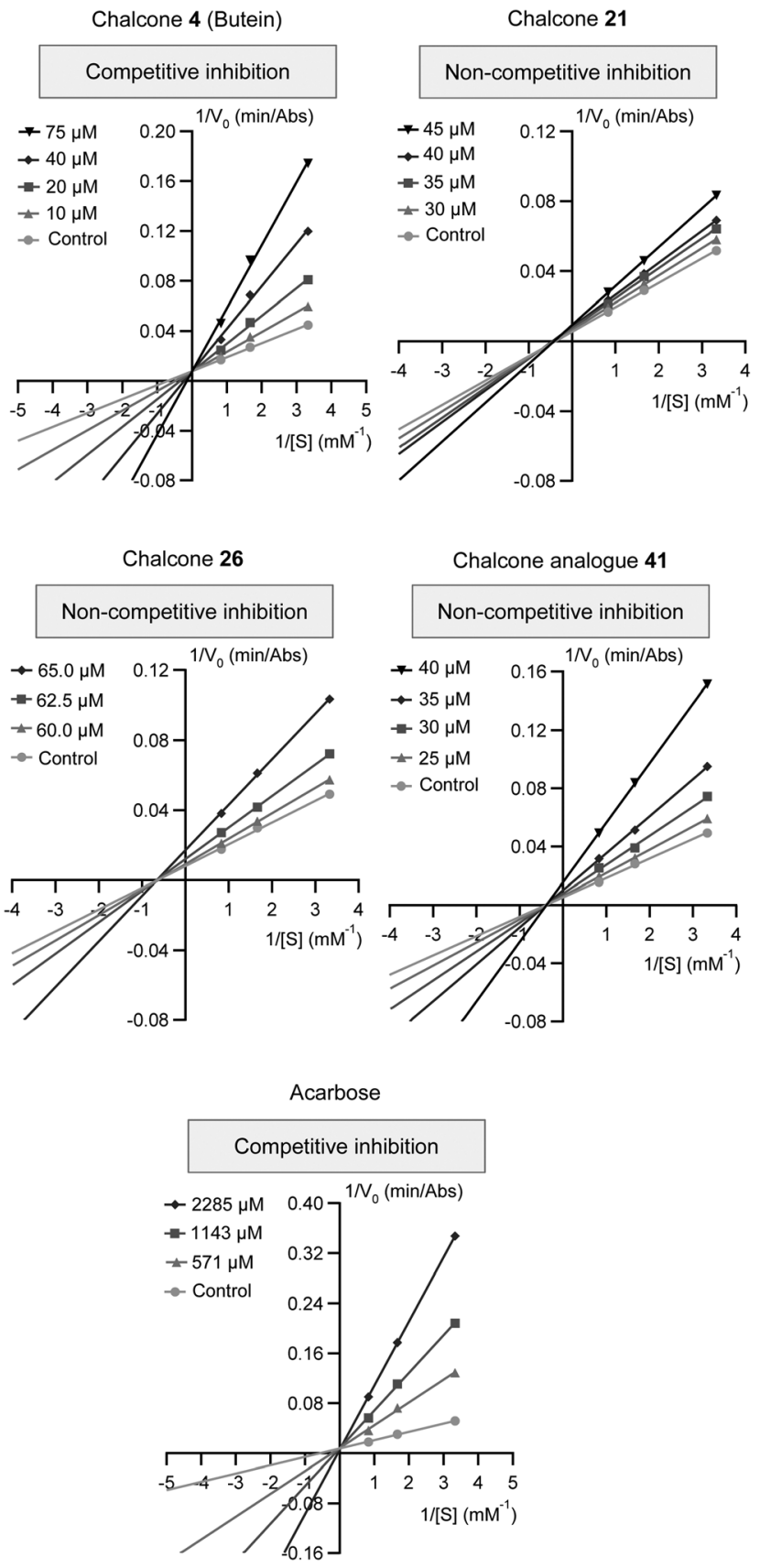

Fig. 4 Lineweaver-Burk plots of $\alpha$-glucosidase inhibition by chalcones 4 (butein), 21, and 26, chalcone analogue 41 and acarbose, the positive control.

\section{Discussion}

The effects of some natural and synthetic chalcones against $\alpha$-amylase and $\alpha$-glucosidase activities have already been studied..$^{20,21,24,26}$ However, several experimental variabilities such as the in vitro model used, the different origins of enzymes and substrates, the diverse origins and concentrations of chalcones, and the time of exposure of the enzymes to the compounds compromise a reliable comparison among studies. The present work overcomes such discrepancies after assessing a panel of forty-one structurally related chalcones and analogues against $\alpha$-amylase and $\alpha$-glucosidase. The inclusion of the unsubstituted inactive chalcone (1) allowed a better understanding of the impact of different substituents on the aryl rings.

Different derivatives regarding the number and type of substituents in the main chalcone scaffold were tested for $\alpha$-amylase inhibitory activity. Chalcones 1,2 , and 3 were studied here for the first time though they did not show any meaningful activity against $\alpha$-amylase. The most active one was the hydroxylated chalcone 4 (butein), where the presence of hydroxy groups at C-2' and C-4' of the A ring and C-3 and C-4 of the $\mathrm{B}$ ring (4, butein) contributed to the inhibitory effect. A simple comparison with chalcone 3 suggests that the presence of the catechol group is crucial for the activity of chalcone 4 (butein). A similar conclusion was drawn by Seo et al. ${ }^{21}$ who studied the inhibitory effects of chalcones substituted with hydroxy, amine and sulfonamide groups. The authors concluded that a catechol group was essential for the observed effect against $\alpha$-amylase. Other works also described the determinant presence of the catechol group on other polyphenol scaffolds, namely in flavonoids. ${ }^{41,42}$

In general, methoxy substituents do not impart favorable inhibitory activity underlined here by the study of chalcones 6-17, tested here for the first time. Chalcone $\mathbf{1 3}$ has two methoxy groups in the $\mathrm{B}$ ring instead of the hydroxyls in chalcone 4 (butein), but with loss in the inhibitory effect. Comparing chalcone 3 with those bearing hydroxy substituents at C-2' and C-4' of the A ring and chalcone $\mathbf{1 2}$ having the same hydroxy substitutions and an additional methoxy substituent at the $\mathrm{B}$ ring, it is possible to conclude that the addition of the methoxy group also did not improve the inhibitory activity of the chalcone. These results are in accordance with Yang et $a{ }^{23}{ }^{23}$ who studied a hydroxylated chalcone and two chalcones bearing a $\mathrm{O}$-glucopyranoside at the A ring. Replacement of the 4-hydroxy group by a methoxy substituent caused an activity decrease. This is consistent with the finding of Bale et al., ${ }^{24}$ who showed that methoxy substituents also decreased the inhibitory activity of the most active chalcone. Nonetheless, the presence of three methoxy substituents at C-3, C-4, and C-5 of the B ring in chalcone 11 slightly improved the inhibitory effect to $36 \pm 3 \%$ at $100 \mu \mathrm{M}$. In spite of the slight inhibitory activity, it suggests that the presence of a 2 '-hydroxy group at the A ring together with methoxy substituents at C-3, C-4 and C-5 of the B ring may benefit the inhibitory activity.

The methyl group is not favorable for the inhibitory effect of chalcones as it is possible to confirm by the lack of activity observed for chalcones 18 and 19. As far as we know, these structures were also studied here for the first time.

Concerning the chalcones holding nitro substituents (20, 21), no reports in literature were found maybe because other groups might have also noticed that this is negligible.

Considering the group of chalcones bearing chloro substituents (22-28), no inhibitory effect was observed. The presence or absence of the 2 '-hydroxy group at the A ring is 
indifferent for the intended effect as it is shown by comparing chalcone 22 with no substitutions at the A ring and a 4-chloro substituent at the B ring with chalcone 25 having a hydroxy group at the A ring and a chloro group at the same position in the $\mathrm{B}$ ring. The effect of the number of chloro substituents at the $\mathrm{B}$ ring was also irrelevant, as concluded comparing the results between chalcone 23 and chalcone 26. A similar pattern of results was obtained by Bale et $a .^{24}$ who also studied chalcones with chloro substituents at the B ring. Despite the significant inhibitory activities of the studied compounds, when the $\mathrm{SCH}_{3}$ group at the B ring was replaced by a chloro substituent on the most active chalcone, the $\mathrm{IC}_{50}$ value increased.

All the tested new chalcone analogues (29-41) showed an inhibitory activity inferior to $20 \%$ up to the highest tested concentrations.

The type of $\alpha$-amylase inhibition by chalcone 4 (butein) and positive control acarbose were determined using the nonlinear regression of Michaelis-Menten with the complement Lineweaver-Burk plots. Since the linear transformations of the Michaelis-Menten equation are less accurate, the type of inhibition and the kinetic constants were also evaluated using Solver supplement of Excel Microsoft Office $^{\mathrm{TM}}$. The two methods were in accordance and showed chalcone $\mathbf{4}$ (butein) as a competitive inhibitor. This means that this compound competes with the substrate for the active sites of the enzyme, thus requiring higher concentrations of CNPG3 to generate the reaction product in the same time window. Acarbose showed a mixed type of inhibition. This means that acarbose can bind to both the free enzyme and the enzyme-substrate complex. This finding is corroborated by other authors. ${ }^{43,44}$ The ANOVA analysis also showed that chalcone $\mathbf{4}$ (butein) and acarbose have a $F$ value higher than the $F$ critical value, which allowed the rejection of the null hypothesis, reinforcing that the compounds exhibited an inhibitory effect on the enzymatic activity.

Concerning $\alpha$-glucosidase, the studies with compounds holding hydroxy groups (2-5) and the hydroxylated and/or methoxylated compounds (6-17), chalcone 4 (butein) was the only chalcone that showed potent inhibitory activity. Negligible activities were found for the remaining hydroxylated and/or methoxylated chalcones $(\mathbf{2}, \mathbf{3}, \mathbf{5}-\mathbf{1 7})$, except chalcone 9 which has a 2'-hydroxy group at the A ring, 3-methoxy and 4-hydroxy substituents at the B ring, which showed a slight inhibitory activity. These results suggest that the presence of the hydroxy groups is crucial for the inhibition of $\alpha$-glucosidase activity. Comparison between chalcone $\mathbf{4}$ (butein) and chalcone 3 provides evidence for the positive effect of the catechol group at C-3 and C-4 of the B ring, which favored the inhibitory effect against $\alpha$-glucosidase activity. The comparison of chalcone 4 (butein) and chalcone 13, bearing hydroxy groups in the same positions of the A ring and methoxy groups at C-3 and C-4 of the B ring, enables us to conclude that the presence of methoxy substituents in the chalcone scaffold also did not bring any advantage for the intended effect. Moreover, in contrast to that of $\alpha$-amylase, increasing the number of methoxy groups in the $\mathrm{B}$ ring did not favor the inhibitory activity which is possible to see through comparison of the derivatives chalcone $\mathbf{1 0}$ and chalcone 11. A similar pattern of results was obtained by Cai et al. ${ }^{26}$ who tested a series of hydroxy and methoxy chalcones against $\alpha$-glucosidase activity. Comparing the tested compounds, chalcones holding methoxy groups showed much lower inhibitory activities than chalcones with hydroxy groups at the same positions. In line with this pattern, Cai et $a .^{26}$ also described that the number and position of the hydroxy substituents in the chalcones is an important factor affecting their inhibitory activity. In fact, the authors also concluded that the presence of a catechol in the A and B rings was relevant for the inhibitory activity. Similar to our results, Ansari et al. ${ }^{27}$ tested one chalcone with a methoxy group at the $\mathrm{B}$ ring, and the $\mathrm{IC}_{50}$ value decreased when a 2'-hydroxy group was added to the A ring of the chalcone. Sun et $a .^{28}$ evaluated the inhibitory activity of hydroxylated, prenylated and/or geranylated chalcones. Replacing hydroxy by methoxy groups of one of the prenylated chalcones, diminished the activity, corroborating our results.

The presence of a nitro group in the chalcone scaffold increased their inhibitory activity against $\alpha$-glucosidase activity. Chalcone analogue $\mathbf{4 1}$ showed almost nine times more activity than the positive control, acarbose. Chalcone 21 exhibited almost seven times more activity than acarbose. The addition of nitro groups to chalcone 2 and chalcone analogue 40, both with a single 2'-hydroxy group at the A ring, resulted in strong inhibitory activities. Moreover, the presence of a hydroxy group was crucial for the $\alpha$-glucosidase inhibition. This is evident by comparing the meaningless result obtained for chalcone 20 with a 4-nitro group at the $\mathrm{B}$ ring with chalcone $\mathbf{2 1}$ bearing an additional 2'-hydroxy group at the A ring. The same effect was observed in chalcone analogues, for example, comparing chalcone analogue 39 bearing a 2-nitro group at the $\mathrm{B}$ ring with chalcone analogue 41 bearing an additional 2'-hydroxy group at the A ring. In turn, changing the position of the nitro group in chalcone 39 to $\mathrm{C}-2^{\prime}$ of the $\mathrm{A}$ ring (chalcone analogue 35) and to $\mathrm{C}-4$ of the $\mathrm{B}$ ring (chalcone analogue 38) did not improve the inhibition of the compounds. As far as we know, chalcone 21 and chalcone analogues bearing nitro groups $(35,38,39,41)$ were studied here for the first time. The inhibitory activity of chalcone $\mathbf{2 0}$ was already evaluated by Ansari et al. ${ }^{27}$ and Jabeen et $a .^{25}$ In both studies, the authors evaluated different substituents, including hydroxy, methoxy, amino and sulfonamido. The less active compound was chalcone $\mathbf{2 0}$.

The derivative $\mathbf{2 6}$ emerged from the chlorochalcones (22-28), herein evaluated for the first time as a potential inhibitor of $\alpha$-glucosidase activity. Recently, Proença et al. ${ }^{39}$ studied a series of chlorinated flavonoids. A flavonoid holding a chloro substituent at the $\mathrm{C}$ ring and hydroxy groups at the $\mathrm{A}$ and $\mathrm{B}$ rings was one of the most active compounds. The same group has shown that chloroflavonoids display potential anti-inflammatory activity, ${ }^{45}$ essential to avoid damages induced by inflammatory processes, which are exacerbated under diabetic 
conditions. These results, together with ours, indicate that this halogen substituent should be included in future works regarding promising chemical structures for the intended effect.

Concerning the other tested compounds, methylchalcones $(18,19)$ were not able to inhibit $\alpha$-glucosidase, up to the highest tested concentration. As far as we know, these chalcones were studied for the first time.

The chalcone analogues with methyl $(30,36)$, methoxy $(31$, 37), chloro (32), fluoro (33), and bromo (34) substituents were not able to inhibit $\alpha$-glucosidase up to the highest tested concentration. As far as we know, these chalcone analogues with two double bonds between the A and B rings were evaluated here for the first time. Cai et al. ${ }^{26}$ studied a series of hydroxylated and methoxylated chalcones and bischalcones which contain two chalcone moieties in a single structure, thus also displaying two double bonds in their structures. The authors reported that the introduction of hydroxy groups in bischalcones were determinant in increasing the inhibitory activity.

By analyzing the results of the $\alpha$-glucosidase inhibition by chalcones, it is possible to observe that their order of potency was: chalcone 4 (butein) $\left(\mathrm{IC}_{50}=21 \pm 2 \mu \mathrm{M}\right)>$ chalcone analogue $41\left(\mathrm{IC}_{50}=41 \pm 1 \mu \mathrm{M}\right)>$ chalcone $21\left(\mathrm{IC}_{50}=53 \pm 1 \mu \mathrm{M}\right)>$ chalcone $26\left(\mathrm{IC}_{50}=87 \pm 3 \mu \mathrm{M}\right)$.

The type of $\alpha$-glucosidase inhibition of the most active compounds was studied using Lineweaver-Burk plots and Solver ${ }^{\mathrm{TM}}$ supplement. Both methods showed that chalcone 4 (butein) and acarbose act via competitive inhibition mechanism. In turn, chalcone 21, chalcone 26, and chalcone 41 showed a non-competitive inhibition. Concerning acarbose, this conclusion is corroborated by other authors. ${ }^{39,43,46}$ However, Son and Lee $^{47}$ reported that acarbose is a mixed type inhibitor. These different results may rely on the experimental conditions used, such as the enzyme and substrate concentrations, the time of interaction between the enzyme and acarbose, and the time of kinetic monitoring of the enzymatic reaction. The ANOVA analysis showed that the compounds have an $F$ value higher than the $F$ critical value which means that despite the variability found in the method precision, the compounds exhibited inhibitory effect against the enzyme activity.

It is currently known that commercially available $\alpha$-glucosidase inhibitors accumulate significantly increased amounts of undigested carbohydrates in the intestine, mainly due to the non-specific inhibition of $\alpha$-amylase, leading to gastrointestinal side effects. A strong $\alpha$-amylase inhibitory activity will lead to prolonged inhibition of starch hydrolysis and the accumulation of undigested carbohydrates in the colon, which will result in excessive bacterial fermentation of carbohydrates that are then used as substrate by bacteria. The consequences for the patients are severe gastrointestinal complications such as diarrhea, flatulence, and abdominal distention. Therefore, inhibitors of $\alpha$-glucosidase with mild inhibitory activity against $\alpha$-amylase, constitute one of major therapeutic strategies for the treatment of type $2 \mathrm{DM}$. Chalcone 4 (butein), the most active compound of this study, showed a moderate inhibition of $\alpha$-amylase and potent inhibition of $\alpha$-glucosidase activity which is important to overcome the side effects of the current therapy. Chalcone 4 (butein) is an important naturally occurring chalcone present in several plants. As far as we know, the antidiabetic activity of butein was already evaluated in vitro and in vivo against aldose reductase, the reductase responsible for the polyol pathway, which converts glucose into sorbitol that accumulates in tissues causing several complications. Butein was shown to significantly reduce the activation of aldose reductase, preventing such complications. ${ }^{48,49}$ Also, it is known that inflammation contributes to the development of insulin resistance and dysfunction of pancreatic $\beta$ cells. Butein was also shown to be a potential therapeutic anti-inflammatory agent. ${ }^{50}$ To the best of our knowledge, the inhibitory activity of butein against $\alpha$-amylase and $\alpha$-glucosidase was studied here for the first time. In our study, chalcone 4 (butein) constitutes the most promising molecule to address type $2 \mathrm{DM}$ control and/or treatment.

\section{Conclusions}

In the present study, a panel of forty-one chalcones and chalcone-type compounds were evaluated for their inhibitory activity of the digestive enzymes, $\alpha$-amylase and $\alpha$-glucosidase, with most of them being tested for the first time, in order to provide deeper insights into their structure and activity. It was possible to conclude that the substitution pattern and the type of substituents influence the inhibitory activity of the compounds, the presence of hydroxy groups being favorable for the intended effect. From the obtained results, it was found that chalcone 4 (butein) was the most active compound showing a moderate inhibition of $\alpha$-amylase and a potent inhibition of $\alpha$-glucosidase. Therefore, the presence of hydroxy groups at C-2' and C-4' of the A ring and at C-3 and C- 4 of the $\mathrm{B}$ ring seems to be important for the inhibitory activity of the studied chalcones. Moreover, it was proved that chalcone 4 (butein) displayed a competitive type of inhibition for both the enzymes.

This study provided important considerations about the chalcones' scaffold and their antidiabetic effect. The obtained results may also contribute for the design of novel molecules that can be useful in the management of type $2 \mathrm{DM}$.

\section{Conflicts of interest}

The authors declare no conflict of interest.

\section{Acknowledgements}

This work received financial support from the European Union (FEDER funds POCI/01/0145/FEDER/007265) and National Funds (FCT/MEC, Fundação para a Ciência e Tecnologia and Ministério da Educação e Ciência) under the Partnership Agreement PT2020 UID/QUI/50006/2013, and "Programa Operacional Competitividade e Internacionalização” 
(COMPETE) (POCI-01-0145-FEDER-029241). Thanks are due to University of Aveiro, Instituto Politécnico de Bragança, FCT/ MEC for the financial support to the QOPNA (FCT UID/QUI/ 00062/2013) and CIMO (UID/AGR/00690/2013) research Units through national funds and where applicable co-financed by the FEDER, within the PT2020 Partnership Agreement, and also to the Portuguese NMR Network. Sónia Rocha acknowledges FCT the financial support for the $\mathrm{PhD}$ grant $(\mathrm{PD} / \mathrm{BD} /$ 145169/2019), in the ambit of "QREN - POPH - Tipologia 4.1 Formação Avançada”, co-sponsored by Fundo Social Europeu (FSE) and by national funds of Ministério da Ciência, Tecnologia e Ensino Superior (MCTES).

\section{References}

1 International Diabetes Federation, IDF Diabetes atlas, 2017.

2 A. Chaudhury, C. Duvoor, V. S. Reddy Dendi, S. Kraleti, A. Chada, R. Ravilla, A. Marco, N. S. Shekhawat, M. T. Montales, K. Kuriakose, A. Sasapu, A. Beebe, N. Patil, C. K. Musham, G. P. Lohani and W. Mirza, Clinical review of antidiabetic drugs: implications for type 2 diabetes mellitus, management, Front. Endocrinol., 2017, 8, 1-12.

3 R. A. DeFronzo, E. Ferrannini, L. Groop, R. R. Henry, W. H. Herman, J. J. Holst, F. B. Hu, C. R. Kahn, I. Raz, G. I. Shulman, D. C. Simonson, M. A. Testa and R. Weiss, Type 2 diabetes mellitus, Nat. Rev. Dis. Primers, 2015, 1, 1-22.

4 American Diabetes Association, Postprandial blood glucose, Diabetes Care, 2001, 24, 775-778.

5 A. Cahn, R. Miccoli, A. Dardano and S. Del Prato, New forms of insulin and insulin therapies for the treatment of type 2 diabetes, Lancet Diabetes Endocrinol., 2015, 3, 638-652.

$6 \mathrm{H}$. Teng and L. Chen, $\alpha$-Glucosidase and $\alpha$-amylase inhibitors from seed oil: A review of liposoluble substance to treat diabetes, Crit. Rev. Food Sci. Nutr., 2017, 57, 34383448.

7 I. A. Brownlee, S. Gill, M. D. Wilcox, J. P. Pearson and P. I. Chater, Starch digestion in the upper gastrointestinal tract of humans, Starch, 2018, 68, 1-12.

8 B. E. Goodman, Insights into digestion and absorption of major nutrients in humans, Adv. Physiol. Educ., 2010, 34, 44-53.

9 D. K. Mahapatra, V. Asati and S. K. Bharti, Chalcones and their therapeutic targets for the management of diabetes: structural and pharmacological perspectives, Eur. J. Med. Chem., 2015, 92, 839-865.

10 R. Tundis, M. R. Loizzo and F. Menichini, Natural products as alpha-amylase and alpha-glucosidase inhibitors and their hypoglycaemic potential in the treatment of diabetes: an update, Mini-Rev. Med. Chem., 2010, 10, 315-331.

11 S. R. Joshi, E. Standl, N. Tong, P. Shah, S. Kalra and R. Rathod, Therapeutic potential of $\alpha$-glucosidase inhibitors in type 2 diabetes mellitus: an evidence-based review, Expert Opin. Pharmacother., 2015, 16, 1959-1981.

12 S. Shahidpour, F. Panahi, R. Yousefi, M. Nourisefat, M. Nabipoor and A. Khalafi-Nezhad, Design and synthesis of new antidiabetic $\alpha$-glucosidase and $\alpha$-amylase inhibitors based on pyrimidine-fused heterocycles, Med. Chem. Res., 2015, 24, 3086-3096.

13 S. L. Gaonkar and U. N. Vignesh, Synthesis and pharmacological properties of chalcones: a review, Res. Chem. Intermed., 2017, 43, 6043-6077.

14 C. Zhuang, W. Zhang, C. Sheng, W. Zhang, C. Xing and Z. Miao, Chalcone: a privileged structure in medicinal chemistry, Chem. Rev., 2017, 117, 7762-7810.

15 Z. Rozmer and P. Perjési, Naturally occurring chalcones and their biological activities, Phytochem. Rev., 2016, 15, 87-120.

16 B. A. Bohm, Introduction to flavonoids - chemistry and biochemistry of organic natural products, Harwood Academic Publishers, Amsterdam, 1999.

17 L. M. Goncalves, I. M. Valente and J. A. Rodrigues, An overview on cardamonin, J. Med. Food, 2014, 17, 633-640.

18 D. I. Batovska and I. T. Todorova, Trends in utilization of the pharmacological potential of chalcones, Curr. Clin. Pharmacol., 2010, 5, 1-29.

19 B. Orlikova, D. Tasdemir, F. Golais, M. Dicato and M. Diederich, Dietary chalcones with chemopreventive and chemotherapeutic potential, Genes Nutr., 2011, 6, 125-147.

20 H. Sun, D. Wang, X. Song, Y. Zhang, W. Ding, X. Peng, X. Zhang, Y. Li, Y. Ma, R. Wang and P. Yu, Natural prenylchalconaringenins and prenylnaringenins as antidiabetic agents: alpha-glucosidase and alpha-amylase inhibition and in vivo antihyperglycemic and antihyperlipidemic effects, J. Agric. Food Chem., 2017, 65, 1574-1581.

21 W. D. Seo, J. H. Kim, J. E. Kang, H. W. Ryu, M. J. CurtisLong, H. S. Lee, M. S. Yang and K. H. Park, Sulfonamide chalcone as a new class of $\alpha$-glucosidase inhibitors, Bioorg. Med. Chem. Lett., 2005, 15, 5514-5516.

22 Y.-C. Hu, Y.-D. Luo, L. Li, M. K. Joshi and Y.-H. Lu, In vitro investigation of 2',4'-dihydroxy-6'-methoxy-3',5'-dimethylchalcone for glycemic control, J. Agric. Food Chem., 2012, 60, 10683-10688.

23 X.-W. Yang, M.-Z. Huang, Y.-S. Jin, L.-N. Sun, Y. Song and H.-S. Chen, Phenolics from Bidens bipinnata and their amylase inhibitory properties, Fitoterapia, 2012, 83, 11691175.

24 A. T. Bale, K. M. Khan, U. Salar, S. Chigurupati, T. Fasina, F. Ali, Kanwal, A. Wadood, M. Taha, S. S. Nanda, M. Ghufran and S. Perveen, Chalcones and bis-chalcones: as potential $\alpha$-amylase inhibitors; synthesis, in vitro screening, and molecular modelling studies, Bioorg. Chem., 2018, 79, 179-189.

25 F. Jabeen, P. V. Oliferenko, A. A. Oliferenko, G. G. Pillai, F. L. Ansari, C. D. Hall and A. R. Katritzky, Dual inhibition of the $\alpha$-glucosidase and butyrylcholinesterase studied by molecular field topology analysis, Eur. J. Med. Chem., 2014, 80, 228-242.

26 C.-Y. Cai, L. Rao, Y. Rao, J.-X. Guo, Z.-Z. Xiao, J.-Y. Cao, Z.-S. Huang and B. Wang, Analogues of xanthones - chalcones and bis-chalcones as $\alpha$-glucosidase inhibitors and anti-diabetes candidates, Eur. J. Med. Chem., 2017, 130, $51-59$. 
27 F. L. Ansari, S. Umbreen, L. Hussain, T. Makhmoor, S. A. Nawaz, M. A. Lodhi, S. N. Khan, F. Shaheen and M. I. Choudhary, Syntheses and biological activities of chalcone and 1,5-benzothiazepine derivatives: promising new free-radical scavengers, and esterase, urease, and alpha-glucosidase inhibitors, Chem. Biodivers., 2005, 2, 487-496.

28 H. Sun, Y. Li, X. Zhang, Y. Lei, W. Ding, X. Zhao, H. Wang, X. Song, Q. Yao, Y. Zhang, Y. Ma, R. Wang, T. Zhu and $\mathrm{P}$. Yu, Synthesis, $\alpha$-glucosidase inhibitory and molecular docking studies of prenylated and geranylated flavones, isoflavones and chalcones, Bioorg. Med. Chem. Lett., 2015, 25, 4567-4571.

29 H. W. Ryu, B. W. Lee, M. J. Curtis-Long, S. Jung, Y. B. Ryu, W. S. Lee and K. H. Park, Polyphenols from Broussonetia papyrifera, displaying potent $\alpha$-glucosidase inhibition, J. Agric. Food Chem., 2010, 58, 202-208.

30 A. Lévai, T. Patonay, A. M. S. Silva, D. C. G. A. Pinto and J. A. S. Cavaleiro, Synthesis of 3-aryl-5-styryl-2-pyrazolines by the reaction of $(E, E)$-cinnamylideneacetophenones with hydrazines and their oxidation into pyrazoles, J. Heterocycl. Chem., 2002, 39, 751-758.

31 A. M. S. Silva, D. C. G. A. Pinto, H. R. Tavares, J. A. S. Cavaleiro, L. Jimeno and J. Elguero, Novel $(E)$ - and $(Z$, )-2-styrylchromones from $(E, E)$-2'-Hydroxycinnamylideneacetophenones - Xanthones from daylight photooxidative cyclization of (E)-2-styrylchromones, Eur. J. Org. Chem., 1998, 2031-2038.

32 D. C. G. A. Pinto, A. M. S. Silva, A. Lévai, J. A. S. Cavaleiro and T. P. J. Elguero, Synthesis of 3-benzoyl-4-styryl-2-pyrazolines and their oxidation to the corresponding pyrazoles, Eur. J. Org. Chem., 2000, 2593-2599.

33 C. M. M. Santos, A. M. S. Silva, J. A. S. Cavaleiro, A. Lévai and T. Patonay, Epoxidation of $(E, E)$-cinnamylideneacetophenones with hydrogen peroxide and iodosylbenzene with salen-MnIII as the catalyst, Eur. J. Org. Chem., 2007, 28772887, DOI: 10.1002/ejoc.200700123.

34 C. Proença, M. Freitas, D. Ribeiro, S. M. Tomé, E. F. T. Oliveira, M. F. Viegas, A. N. Araújo, M. J. Ramos, A. M. S. Silva, P. A. Fernandes and E. Fernandes, Evaluation of a flavonoids library for inhibition of pancreatic $\alpha$-amylase towards a structure-activity relationship, J. Enzyme Inhib. Med. Chem., 2019, 34, 577-588.

35 R. M. Bezerra, I. Fraga and A. A. Dias, Utilization of integrated Michaelis-Menten equations for enzyme inhibition diagnosis and determination of kinetic constants using Solver supplement of Microsoft Office Excel, Comput. Methods Programs Biomed., 2013, 109, 26-31.

36 A. A. Dias, P. A. Pinto, I. Fraga and R. M. F. Bezerra, Diagnosis of enzyme inhibition using Excel Solver: a combined dry and wet laboratory exercise, J. Chem. Educ., 2014, 91, 1017-1021.

37 F. J. Burguillo, A. J. Wright and W. G. Bardsley, Use of the F test for determining the degree of enzyme-kinetic and ligand-binding data. A Monte Carlo simulation study, Biochem. J., 1983, 211, 23-34.
38 K. Yamaoka, T. Nakagawa and T. Uno, Application of Akaike's information criterion (AIC) in the evaluation of linear pharmacokinetic equations, J. Pharmacokinet. Biopharm., 1978, 6, 165-175.

39 C. Proença, M. Freitas, D. Ribeiro, E. F. T. Oliveira, J. L. C. Sousa, S. M. Tomé, M. J. Ramos, A. M. S. Silva, P. A. Fernandes and E. Fernandes, alpha-Glucosidase inhibition by flavonoids: an in vitro and in silico structureactivity relationship study, J. Enzyme Inhib. Med. Chem., 2017, 32, 1216-1228.

40 O. C. Miles and L. Main, Kinetics and Mechanism of the Cyclisation of 2',6'-Dihydroxy-4,4'-dimethoxychalcone; Influence of the 6'-Hydroxy Group on the Rate of Cyclisation under Neutral Conditions, J. Chem. Soc., Perkin Trans. 2, 1985, 1639-1642, DOI: 10.1039/P29850001639.

41 J. Xiao, X. Ni, G. Kai and X. Chen, A review on structureactivity relationship of dietary polyphenols inhibiting alpha-amylase, Crit. Rev. Food Sci. Nutr., 2013, 53, 497-506.

$42 \mathrm{H}$. Cao and X. Chen, Structures required of flavonoids for inhibiting digestive enzymes, Anticancer Agents Med. Chem., 2012, 12, 929-939.

43 M. J. Kim, S. B. Lee, H. S. Lee, S. Y. Lee, J. S. Baek, D. Kim, T. W. Moon, J. F. Robyt and K.-H. Park, Comparative study of the inhibition of $\alpha$-glucosidase, $\alpha$-amylase, and cyclomaltodextrin glucanosyltransferase by acarbose, isoacarbose, and acarviosine-glucose, Arch. Biochem. Biophys., 1999, 371, 277-283.

44 S. H. Yoon and J. F. Robyt, Study of the inhibition of four alpha amylases by acarbose and its $4 \mathrm{IV}-\alpha-$ maltohexaosyl and $4 \mathrm{IV}-\alpha-$ maltododecaosyl analogues, Carbohydr. Res., 2003, 338, 1969-1980.

45 M. Freitas, D. Ribeiro, S. M. Tomé, A. M. S. Silva and E. Fernandes, Synthesis of chlorinated flavonoids with anti-inflammatory and pro-apoptotic activities in human neutrophils, Eur. J. Med. Chem., 2014, 86, 153-164.

46 N. Chatsumpun, B. Sritularak and K. Likhitwitayawuid, New biflavonoids with $\alpha$-glucosidase and pancreatic lipase inhibitory activities from Boesenbergia rotunda, Molecules, 2017, 2, 1-13.

$47 \mathrm{H}$. Son and S. H. Lee, Comparison of $\alpha$-glucosidase inhibition by Cudrania tricuspidata according to harvesting time, Biomed. Rep., 2013, 1, 624-628.

48 E. H. Lee, D.-G. Song, J. Y. Lee, C.-H. Pan, B. H. Um and S. H. Jung, Inhibitory effect of the compounds isolated from Rhus verniciflua on aldose reductase and advanced glycation endproducts, Biol. Pharm. Bull., 2008, 31, 16261630.

49 S. S. Lim, S. H. Jung, J. Ji, K. H. Shin and S. R. Keum, Synthesis of flavonoids and their effects on aldose reductase and sorbitol accumulation in streptozotocininduced diabetic rat tissues, J. Pharm. Pharmacol., 2001, 53, 653-668.

50 G. Padmavathi, N. K. Roy, D. Bordoloi, F. Arfuso, S. Mishra, G. Sethi, A. Bishayee and A. B. Kunnumakkara, Butein in health and disease: A comprehensive review, Phytomedicine, 2017, 25, 118-127. 